\section{Gum Yield as Affected by Capsule Age, Firmness, Gum Collecting Methods, and Phenotypes in Opium Poppy}

\author{
Mary C. Acock, Zhongchun Wang, Basil Acock, and Robert Jones \\ U.S. Department of Agriculture, Agriculture Research Service, Systems Remote \\ Sensing and Modeling Laboratory, Beltsville, MD 20705
}

Additional index words. opium poppy, yield

\begin{abstract}
The U.S. State Dept. annually publishes estimates of narcotic drug crop production worldwide. The areas under cultivation are well known but yields per unit land area are not. Determining opium gum yield from illicitly grown poppy Papaver somniferum $\mathrm{L}$. is difficult and dangerous. Removing plants from the field and harvesting gum in a safe place would allow us to measure gum yield from one short field visit. To interpret these results in terms of total gum yield from the field, one must know how the measured gum is affected by gum collecting method, capsule age, and phenotype. Opium poppy seeds from three phenotypes (purple, white, and red-white flowers) were grown in a greenhouse and plants were either cut at the soil level or left intact for opium gum harvest at 7, 12, and 22 days after flowering (DAF). Capsule firmness was measured to estimate gum yield and capsule age, and the relationship between total gum yield and yield from the first lancing was examined. The average gum yield $\left(8.4 \mathrm{mg} \cdot \mathrm{g}^{-1} \mathrm{dry}\right.$ weight capsule) for the purpleflowered phenotypes was $17 \%$ and $25 \%$ lower than for the white- and red/white-flowered phenotypes, respectively. Capsule firmness of the three phenotypes varied from $\approx \mathbf{8 0 0}$ to $2300 \mathrm{~N} \cdot \mathrm{m}^{-1}$ as the capsule aged. Gum yield and capsule firmness increased with capsule age but the timing of those changes differed among phenotypes. No significant correlations were found between capsule firmness and gum yield or between capsule firmness and age. Therefore, capsule firmness cannot be used to predict gum yield or capsule age. Gum yield from the first lancing was linearly correlated with total gum yield $\left(\mathbf{r}^{2}=0.82\right)$. Since this relationship changes with growing condition, it is insufficient to predict total gum yield. Gum yield from cut plants was significantly lower than from intact plants for all three phenotypes at 22 DAF and for white-flowered phenotypes at $12 \mathrm{DAF}$. No difference in gum yield was observed between cut and intact plants at 7 or 12 DAF for purple and red/whiteflowered phenotypes. The relationship between gum yield from cut and intact plants was too variable to predict gum yield from intact plants by measuring gum yield from cut plants.
\end{abstract}

The U.S. State Dept. has been charged by Congress with annually estimating narcotic drug crop production for all countries that are illegal producers. These data are used to make decisions about foreign aid and the allocation of resources to the War on Drugs. Areas under cultivation are known and the U.S. Dept. of Agriculture, Agricultural Research Service (USDA-ARS), has been asked to develop methods for estimating yields per unit land area. Estimating opium gum yield of poppy (Papaver somniferum L.) plants in the field is difficult because most crops are illegal and are grown in areas where the government has little control. It is, therefore, dangerous to visit a poppy field, and especially dangerous to visit it more than once. Normally, gum is harvested

Received for publication 7 Sept. 1995. Accepted for publication 18 July 1996. Mention of a proprietary product does not imply its approval to the exclusion of other products that may also be suitable. This paper has been cleared by the Public Affairs Office of the U.S. Dept. of State. The cost of publishing this paper was defrayed in part by the payment of page charges. Under postal regulations, this paper therefore must be hereby marked advertisement solely to indicate this fact. to predict gum yield? 4) Can total gum yield be estimated by measuring gum from one lancing? and 5) Do these relationships depend on phenotype?

\section{Materials and Methods}

Plant materials and growth conditions. The study was performed in a greenhouse at the Beltsville Agricultural Research Center in Beltsville, Md. (lat. $\left.39^{\circ} \mathrm{N}\right)$. Opium poppy seeds of three phenotypes (purple, white, and red/ white flowers) from Thailand were sown in 4L pots on 1 Sept. 1992. The growing medium consisted of $0.5 \mathrm{~m}^{3}$ vermiculite and $0.5 \mathrm{~m}^{3}$ sphagnum peat amended with $4.03 \mathrm{~kg}$ dolomitic lime, $5.57 \mathrm{~kg}$ of a 6-month slow-release fertilizer containing $14 \mathrm{~N}-6.1 \mathrm{P}-11.6 \mathrm{~K}$, and $1.06 \mathrm{~kg}$ Micromax containing $\left(\mathrm{g} \cdot \mathrm{kg}^{-1}\right) 120 \mathrm{~S}$, $120 \mathrm{Fe}, 25 \mathrm{Mn}, 10 \mathrm{Zn}, 5 \mathrm{Cu}, 1 \mathrm{~B}$, and $0.5 \mathrm{Mo}$. The seedlings were thinned to one plant per pot, and grown under natural light with a supplement of tungsten lights that extended the day length to $13 \mathrm{~h}$. The thermostat in the greenhouse was set to maintain air at 20C. The seedlings were watered twice daily, and fertilized with Peters' water soluble $20 \mathrm{~N}-8.6 \mathrm{P}-$ $16.6 \mathrm{~K}$ fertilizer at a $\mathrm{N}$ concentration of 100 $\mathrm{mg} \cdot \mathrm{L}^{-1}$.

At 7, 12, and 22 days after flowering (DAF), the capsules were lanced, and gum yield, plant organ dry weight, and capsule firmness were measured on intact and cut plants for each phenotype. The capsule was weighed after lancing; therefore, capsule dry weight did not include any gum collected. Since the gum dry weight averaged $<1 \%$ of the capsule dry weight, the amount of gum had little effect on overall capsule dry weight.

Opium gum collection. The technique of opium gum collection was similar to that described by Gupta (1984). A three-bladed knife was used to make longitudinal cuts, $2 \mathrm{~mm}$ apart into the capsule wall. Each cut was $\approx 2 \mathrm{~cm}$ long and $1 \mathrm{~mm}$ deep. At each lancing, three knife cuts were made, resulting in a total of nine longitudinal cuts. The milky latex that exuded from the cuts dried to a dark brown gum overnight and was scraped off the capsule the following day onto pre-weighed foil. The gum was then dried in an oven at $80 \mathrm{C}$ for $48 \mathrm{~h}$ and weighed. On cut plants, those used to simulate plants taken in a single field visit and transported back to safety, the shoots of plants were cut off at soil level, placed in black plastic bags, and left in the greenhouse overnight. Then, the cut ends of the stems were immersed in water for 1 day to allow the plants to regain turgidity. On the following day, the terminal capsules were lanced.

Capsule firmness measurements. The firmness of the capsule was measured with a firmness meter using the method described by Timm et al. (1993). Essentially, the meter uses a caliper, driven by a stepping motor, to squeeze the object of interest. A force transducer on the caliper measures force vs. deformation. For each measurement, a capsule was placed between the plates of the firmness meter with about $1 \mathrm{~mm}$ of clearance. The capsule was squeezed up to a threshold force of 3.92 New- 
Table 1. Opium gum dry weight ( $\mathrm{mg} \cdot \mathrm{g}^{-1}$ dry weight terminal capsule) of cut and intact poppy plants at 7,12 , and 22 days after flowering for purple, white, and red/white-flowered phenotypes.

\begin{tabular}{lccr}
\hline & \multicolumn{3}{c}{ Opium gum dry weight $\left(\mathrm{mg} \cdot \mathrm{g}^{-1}\right)$} \\
\cline { 2 - 4 } & \multicolumn{3}{c}{ Days after flowering } \\
\cline { 2 - 4 } Phenotype & 7 & 12 & 22 \\
\hline Purple & 8.87 & 7.60 & 8.35 \\
Cut & 6.42 & 5.84 & $* 3$ \\
Intact & $\mathrm{NS}$ & $\mathrm{Ns}$ & \\
Cut vs. intact & & 3.70 & 9.56 \\
White & 5.61 & 7.55 & $* 37$ \\
Cut & 4.85 & $*$ & $*$ \\
Intact & $\mathrm{NS}$ & & 7.33 \\
Cut vs. intact & & 13.06 & 15.21 \\
Red/white & 7.33 & 11.50 & $*$ \\
Cut & 10.55 & $\mathrm{NS}$ & \\
Intact & $\mathrm{NS}$ & & \\
Cut vs. intact & & & \\
\hline
\end{tabular}

$\overline{\mathrm{NS},}$ "Nonsignificant or significant at $P \leq 0.05$, respectively.

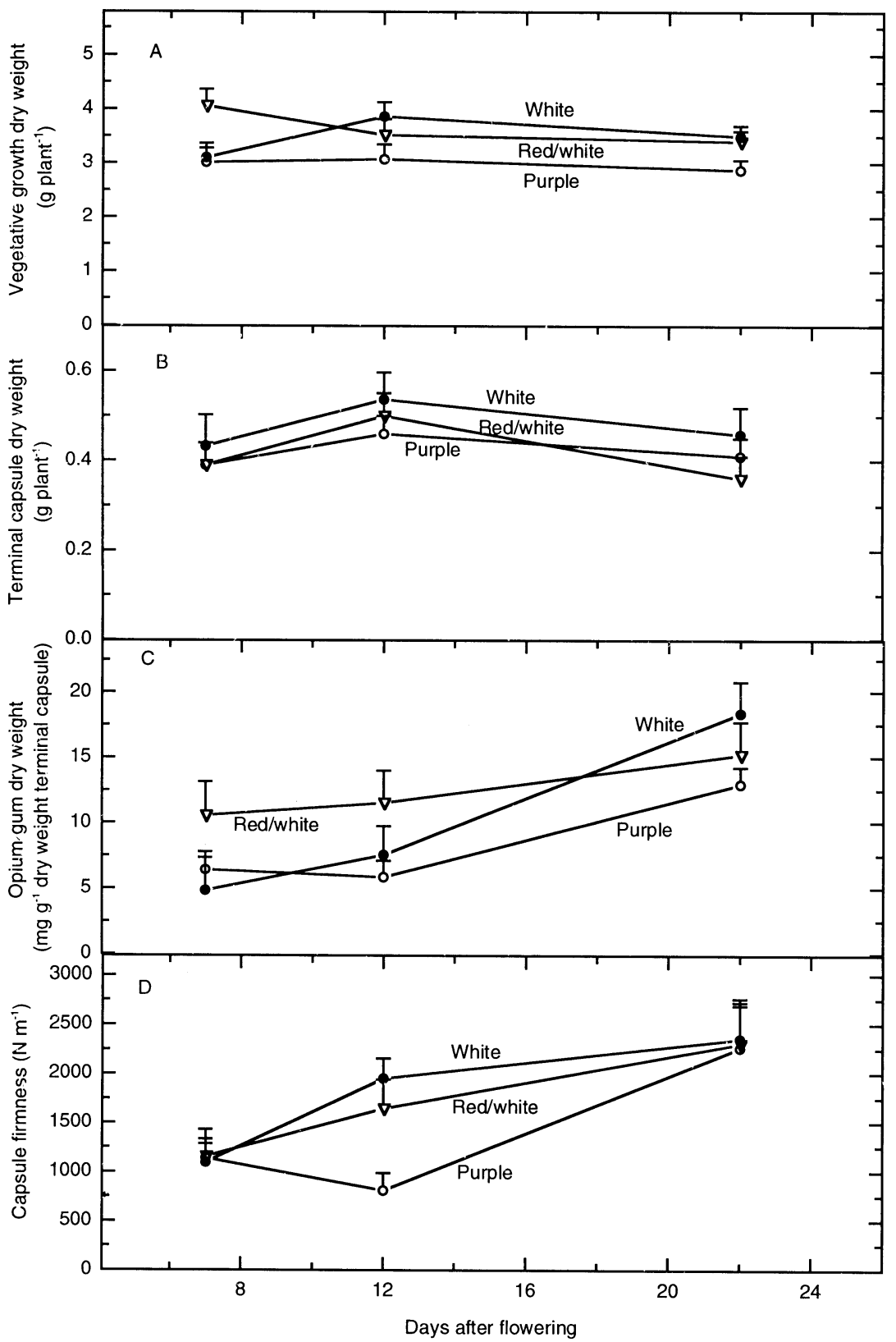

Fig. 1. Vegetative growth and capsule dry weight, opium gum yield, and capsule firmness at 7, 12, and 22 days after flowering in purple, white, and red/white-flowered poppy plants (intact plants only). Vegetative growth included stems and leaves of the plants. Vertical bars represent standard errors $(\mathrm{n}=10)$. tons $(\mathrm{N})$, the maximum force that had been tested and set before the measurements. Firmness components and force vs. deformation curve were recorded and stored as files in a portable computer connected to the firmness meter. The slope of the compression curve was used as the measure of capsule firmness. Calibration of the force transducer was made before use and periodically during use by measuring the signal output for no weight and for a 100 -g weight on the loading plate.

Estimation of total gum yield from first lancing. To estimate the total gum yield from a single lancing for all three phenotypes, a separate treatment was added to the experiment. In this treatment, capsules were lanced three times at 3 -day intervals beginning 7 days after flowering. The total gum yield was calculated as the sum of the three lancings.

Experiment design and statistical analysis. Treatments were arranged in a factorial randomized complete-block design with three phenotypes, three harvest dates, and two gum collecting methods (cut and intact plants). There were 10 single-plant replications per treatment combination. The separate treatment described earlier was added to the block design. Plants were blocked according to size just before treatments were begun. Analysis of variance was performed using JMP version 2.0 (SAS Inst., Cary, N.C.). The relationship between capsule firmness and gum yield, and between first lancing and total gum yield was analyzed as a linear dependence of the second variable on the first.

\section{Results and Discussion}

Gum yield from cut vs. intact plants. Gum dry weight from intact plants varied from 4.9 to $18.4 \mathrm{mg} \cdot \mathrm{g}^{-1}$ dry weight of terminal capsule for capsules at 7 to 22 DAF (Table 1). Gum yield was not significantly different between cut and intact plants at 7 DAF for any of the three phenotypes. At $12 \mathrm{DAF}$, the ratio of gum from intact : cut plants was 2.0 for the whiteflowered phenotypes. No statistical difference in gum yield between cut and intact plants was found for the other two phenotypes at 12DAF. At $22 \mathrm{DAF}$, the ratios of gum from intact : cut plants were 1.5 for purple, 1.9 for white, and 2.1 for the red/white.

Our results indicate that the relationship between gum from intact and cut plants changes with capsule age and phenotype. To use cut plants to estimate gum yield from intact plants would require demonstrated consistency of the relationship between cut and intact plant gum yields or some means of measuring the influence of other variables on this relationship.

Gum yield by phenotype. The red/whiteflowered phenotypes had a higher gum yield than the purple-flowered phenotypes at 7 and 12 DAF, while the white-flowered phenotypes had a higher gum yield than the purpleflowered at 22 DAF (Fig. 1C). The average gum yields in terms of unit terminal capsule dry weight for the red/white- and white-flowered phenotypes were $25 \%$ and $17 \%$ higher than the purple-flowered plants, respectively. 


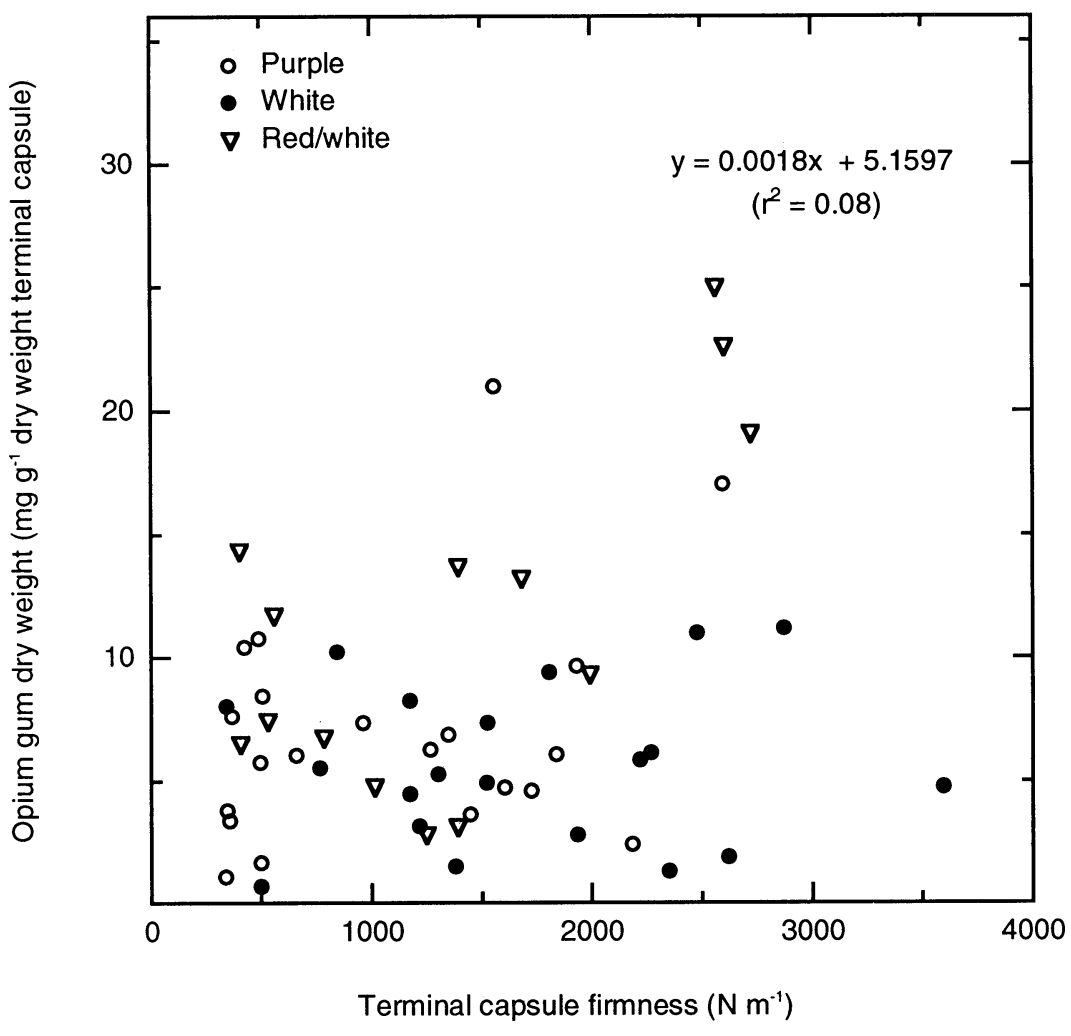

Fig. 2. Correlation between capsule firmness and opium gum yield of intact poppy plants. Data were combined over 7, 12, and 22 days after flowering for purple, white, and red/white-flowered phenotypes.

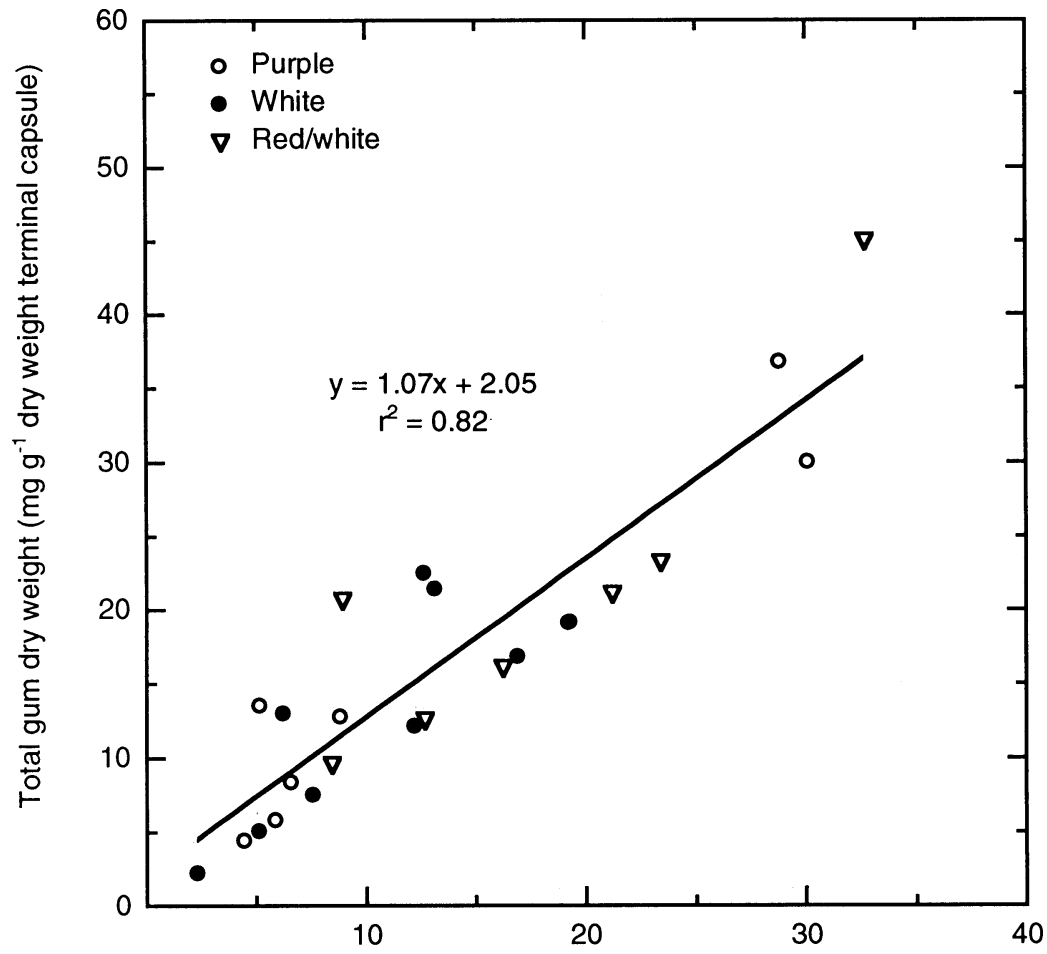

First lancing gum dry weight ( $\mathrm{mg} \mathrm{g}^{-1}$ dry weight terminal capsule)

Fig. 3. Correlation between opium gum yield from first lancing and total gum yield in purple, white, and red/ white-flowered plants. Capsules were lanced three times at 3-day intervals. The first lancing was performed at 7 days after flowering and the total gum yield was the sum of the three lancings.
Significant varietal differences in gum yield have been found by others (Jain et al., 1990; Nigam and Rawat, 1984; Sethi et al., 1990). Results of this experiment indicate that phenotypes not only differ in the total amount of gum yield, but also in the pattern of gum yield as a function of capsule age.

Changes in gum yield with capsule age. Gum yield of the intact plants increased slightly from 7 to $12 \mathrm{DAF}$ for the white- and red/whiteflowered phenotypes but decreased for the purple-flowered phenotypes (Fig. 1C). Gum yield increased dramatically from 12 to 22 DAF for all three phenotypes. The gum dry weights at 22 days were $\approx 1.5,2.0$, and 3.8 times higher than those at 7 days for red/white, purple, and white-flowered plants, respectively. The increase in gum dry weight from 12 to 22 DAF could not be attributed to increased capsule dry weight, since capsule dry weight of the three phenotypes was either the same or lower at 22 DAF than samples taken at $12 \mathrm{DAF}$ (Fig. 1B). The gum must have been synthesized in the capsules or translocated into them.

Differences in opium gum yield attributed to capsule age have been documented (e.g., Annett, 1920). Our results indicated that gum yield was highest at $22 \mathrm{DAF}$, the last harvest we made, when compared with 7 or 12 DAF. The time of maximal capsule dry weight or capsule gum yield may vary, primarily depending on growing conditions (Bunting, 1963). Our results showed that maximum gum yield was not coincident with the maximum dry weight of the capsule but occurred some time later (Fig. 1B, 1C).

Changes in capsule firmness with age. The capsule generally became harder from 7 to 22 DAF except that firmness in the purple-flowered phenotypes did not increase until sometime after 12 DAF (Fig. 1D). All three phenotypes had similar values $\left(\approx 1100 \mathrm{~N} \cdot \mathrm{m}^{-1}\right)$ for capsule firmness at 7 DAF but the purpleflowered poppy had a significantly lower firmness reading than the other two phenotypes at 12 DAF. The capsule firmness in all three phenotypes increased to similar values $(\approx 2300$ $\mathrm{N} \cdot \mathrm{m}^{-1}$ ) by $22 \mathrm{DAF}$. Although capsules were firmest at 22 DAF for all phenotypes, we were unable to predict gum yield (Fig. 2) or capsule age from this reading.

Relationship between first lancing and total gum yield. A high linear correlation $(\mathrm{r}=$ 0.91 ) between first lancing and total gum yield of three lancings existed and the correlations were similar for all three phenotypes (Fig. 3). The average first lancing yielded $80 \%$ of total gum yield. However, these greenhouse results do not compare well with those obtained from field studies in Thailand, where the first lancing accounted for $\approx 40 \%$ of the total gum yield (USDA-ARS, Systems Research Laboratory, 1992). Also, gum yield percentages of first lancings have been found to vary between years (Turkhede and Singh, 1981), ranging from $39 \%$ to $68 \%$. The main reason for the high gum yield percentage of the first lancing in this study was that many plants only produced gum from the first lancing. Variation from experiment to experiment is too great to generalize a specific relationship between first 
lancing and total gum yield. To be useful as an estimator, the percentage of gum in the first lancing must either be stable over the normal range of growing conditions or must be predictable from knowing growing conditions. Our data were obtained from plants grown in a greenhouse over winter in which plants were induced to flower early, resulting in small plants and a high percentage of total gum yield in the first lancing. The relationship needs to be evaluated under other known growing conditions.

In conclusion, gum yield varies with phenotype and capsule age. The relationship between gum yield from the cut plants and intact plants also changes with capsule age and phenotype. Therefore, estimating gum yield of intact plants from gum yield of cut plants requires knowledge of capsule age and pheno- type. We were unable to predict capsule age from firmness data because it was too variable. Since capsule firmness was measured only three times and for a relatively short period (721 DAF), more detailed studies on capsule age and firmness are necessary before one can determine whether capsule firmness can be used to predict capsule age.

\section{Literature Cited}

Annett, H. E. 1920. Factors influencing alkaloidal content and yield of latex in the opium poppy (Papaver somniferum). Memoirs Dept. Agr. India 5:619-636.

Bunting, E.S. 1963. Changes in the capsule of $\mathrm{Pa}$ paver somniferum between flowering and maturity. Ann. Appl. Biol. 51:459-471.

Gupta, R. 1984. Improved production methods for opium poppy. Indian Hort. 28:9-26.
Jain, P.M., B.L. Gaur, and P.C. Gupta. 1990. Response of opium poppy varieties to nitrogen. Indian J. Agron. 35:243-245.

Nigam, K.B. and G.S. Rawat. 1984. Stability of performance of opium-poppy varieties over years. Indian J. Agr. Sci. 54:771-773.

Sethi, K.L., R.L. Sapra, R. Gupta, K.S. Dhindsa, and N.K. Sangwan. 1990. Performance of poppy cultivars in relation to seed, oil and latex yields under different environments. J. Sci. Food Agr. 52:309-313.

Timm, E.J., P.R. Armstrong, G.K. Brown, and R.M. Beaudry. 1993. A portable instrument for measuring firmness of cherries and berries. Amer. Soc. Agr. Eng., Paper No. 93-6539.

Turkhede, B.B. and R. Singh. 1981. Effect of number and methods of lancing on yield and quality of opium poppy. Indian J. Agron. 26:461-462.

U.S. Department of Agriculture. 1992. Thailand opium yield project (1991-1992). U.S. Dept. Agr., Agr. Res. Serv., Systems Res. Lab. 